\title{
Low-Complexity Spectral Partitioning Based MUSIC Algorithm for Automotive Radar
}

\author{
Sangdong Kim ${ }^{1}$, Bong-Seok Kim ${ }^{1}$, Yeonghwan $\mathrm{Ju}^{1}$, Jonghun Lee ${ }^{1}$ \\ ${ }^{I}$ Advanced Radar Technology Laboratory, Convergence Research Center for Future Automotive \\ Technology, Daegu Gyeongbuk Institute of Science \& Technology, \\ Daegu, Republic of Korea \\ jhlee@dgist.ac.kr
}

\begin{abstract}
This paper proposes a low-complexity spectral partitioning (SP) based MUSIC algorithm for automotive radar. For short-range radar (SRR), the range accuracy and resolution of $24 \mathrm{GHz}$ ISM band radar should be improved because the requirements of automotive sensing become more demanding over time. To improve the performance of range estimation, high resolution based algorithms such as the estimation of signal parameters via rotational invariance techniques (ESPRIT) and multiple signal classification (MUSIC) have been proposed. However, in a low signal-tonoise ratio (SNR), the high resolution algorithm shows degraded performance to estimate the parameters. To solve this problem, SP based high resolution algorithms have been studied recently. However, for real-time operation, the conventional SP method cannot be applied to automotive radar due to its high complexity. Therefore, a low complexity SP based MUSIC is designed to maintain the performance of the range accuracy and resolution in a low SNR environment. While the complexity of the proposed algorithm is less than the SP-MUSIC, Monte-Carlo simulation results and experimental results show that the estimation performance of the proposed method is similar to that of SP-MUSIC in terms of various parameters.
\end{abstract}

Index Terms-Vehicle radar; spectrum partitioning; MUSIC; low SNR; low complexity.

\section{INTRODUCTION}

Driver assistance systems are one of the major applications to create a safe and convenient automotive environment. Active or passive driver assistance systems should recognize the stationary and moving target around vehicles. Given this, many kinds of sensors such as radar, lidar, and camera are needed [1]. In particular, millimeterwave radar is useful in many automotive applications such as adaptive cruise control (ACC) and lane change assistance (LCA) regardless of bad weather such as rain and fog for detecting long and short range targets [2], [3]. Since automotive requirements are gradually increasing, automotive radar needs improved range resolution and maximum distance. With the conventional $24 \mathrm{GHz}$ ISM band radar with bandwidth of $200 \mathrm{MHz}$ and a maximum distance of $30 \mathrm{~m}$, it is difficult to meet various requirements

Manuscript received 21 January, 2017; accepted 12 June, 2017.

This work was supported by the DGIST R\&D Program of the Ministry of Science, ICT and Future Planning, Korea (17-IT-01). of automotive sensing. Over the past decades, to improve the performance of range resolution, high resolution based algorithms such as the estimation of signal parameters via rotational invariance techniques (ESPRIT) [4], multiple signal classification (MUSIC) [5], and the autoregressive method [6] have been proposed. In the case of low SNR, a high resolution algorithm that exploits spectral partitioning (SP) [7] has been developed to improve the estimation performance. The SP based high resolution algorithm divides the spectrum into multiple spectrum sub-bands and a high resolution algorithm is generated using each sub-band signal. However, the SP based high resolution algorithm cannot be adopted for real-time implementation due to the high computational load. Therefore, in this paper, in order to satisfy the required accuracy requirements and reduce complexity compared with the conventional estimator, we propose a low-complexity SP based MUSIC algorithm, which is the most widely used algorithm, for vehicle FMCW radar.

\section{SIGNAL MODEL}

The mathematical form of the transmitted FMCW signal for automotive radar is defined as

$$
s(t)=\left\{\begin{array}{cc}
\exp \left[j\left(\omega_{s} t+(\mu / 2) t^{2}\right)\right], & \text { for } 0 \leq t<T_{\text {sym }}, \\
0, & \text { elsewhere }
\end{array}\right.
$$

where $\omega_{s}$ is the initial frequency, $\mu$ is the rate of change of the instantaneous frequency of a chirp symbol, $\mu=\omega_{B W} / T_{\text {sym }}$, $\omega_{B W}$ is the bandwidth of the FMCW signal, and $T_{\text {sym }}$ is the signal period of the FMCW symbol.

Consider $M$ targets in the road environment, and let $\tau_{m}$ denote the round-trip time delay of the $m$-th target. Then, assuming a time-invariant channel over $T_{\text {sym }}$, the received signal can be represented by

$$
y(t)=\sum_{m=0}^{M-1} a_{m} s\left(t-\tau_{m}\right)+\omega_{\mathrm{n}}(t)
$$

where $a_{m}$ denotes the complex amplitude for the $m$-th target and $\omega_{n}(t)$ is the additive white Gaussian noise (AWGN) 
signal. In the received part, de-chirping is defined as the multiplication of the received FMCW signals $y(t)$ and the conjugation of the FMCW transmitted signals $s^{*}(t)$ such that

$$
r(t)=y(t) \times s^{*}(t)
$$

where $r(t)$ indicates that $y(t)$ is transformed into a sinusoidal form. Through (3), it is derived that the transformed signals $r(t)$ have a sinusoidal waveform such that

$$
r(t)=\sum_{m=0}^{M-1} a_{m} \exp \left[j\left(\mu \tau_{m} t+\omega_{s} \tau_{m}-\mu / 2 \tau_{m}{ }^{2}\right)\right]+\omega_{\mathrm{n}}(t) .
$$

When the Nyquist sampling frequency $f_{s}=1 / T_{s}$ is given, the discrete time model $r[n]$ of $r(t)$, which is received through an analog-to-digital converter (ADC), can be described as follows

$$
r[n]=\sum_{m=0}^{M-1} a_{m} \exp \left[j\left(\mu \tau_{m} T_{s} n+\omega_{s} \tau_{m}-\mu / 2 \tau_{m}{ }^{2}\right)\right]+\omega[n]
$$

where $n=0,1, \ldots N-1$.

\section{PROPOSED LOW-COMPLEXITY SPECTRAL PARTITIONING BASED MUSIC ALGORITHM FOR AUTOMOTIVE RADAR}

In order to reduce noise effects for automotive radar, the proposed algorithm presents a low complexity SP based MUSIC algorithm that detects the signal region among multiple spectral sub-bands. The proposed method is composed of a signal-based spectral partitioning part and an efficient spectrum generator part. Through the proposed algorithm, we can obtain MUSIC results that are robust to low SNR for a low complexity system.

\section{A. Signal-based SP Method for SNR Improvement}

The proposed low complexity SP-MUSIC method for SNR improvement is shown in Fig. 1. The signal-based SP block of the proposed method is composed of DFT, windowing, a comparator, and a signal selector with a switch. The DFT is the traditional method to detect and estimate multiple signals. The DFT output is accomplished as follows

$$
R[l]=\sum_{n=0}^{N-1} r[n] e^{-j 2 \pi l n / N} \text { for } l=0,1, \ldots, N-1,
$$

where $l$ denotes an index of the discrete frequency for the estimation of the time delay. After the spectrum can be divided into $S$ sub-bands of size $B=[N / S]$ spectral samples using windowing, where the notation $[x]$ represents the smallest integer larger than $x$, we compute the comparator block using windowing DFT results and threshold signal. We can then obtain the signal-based spectrum index vector $\boldsymbol{I}_{\boldsymbol{t}}=$ $\left[I_{1}, I_{2}, \ldots, I_{M}\right]$ in the case where $M$ multiple targets exist through the signal selector block. In order to achieve the sub-band of interest, let us define a spectral partition matrix for the $s$-th sub-band $s=0,1,2, \ldots, S$-1, such as

$$
\mathbf{P}_{s}=\operatorname{diag}\left(p_{0}^{s}, p_{1}^{s}, \ldots, p_{N-1}^{s}\right)
$$

where the diagonal element $p_{i}^{s}$ is given by

$$
p_{i}^{S}=\left\{\begin{array}{ll}
1 & \mathrm{i} \in \mathbf{L}_{M} \\
0 & \text { otherwise }
\end{array},\right.
$$

where, $\boldsymbol{L}_{M}$ is a set of indexes about selected window signal among total sub-band [7] such as

$$
\begin{gathered}
\mathbf{L}_{M}=\left[l_{i, j}\right]_{j=0}^{B-1} \text { with } l_{i, j}=((i-1) B+j) \\
\text { for } i=I_{1}, I_{2}, \ldots, I_{M}
\end{gathered}
$$

After signal-based windowing is achieved such as

$$
\mathbf{R}_{w, s}=\mathbf{P}_{s} \mathbf{R},
$$

where

$$
\mathbf{R}=[R[0], R[1], \ldots, R[N-1]]^{\mathrm{T}}
$$

$\mathbf{R}_{\mathrm{w}, \mathrm{s}}=\left[R_{\mathrm{w}, \mathrm{s}}[0], R_{\mathrm{w}, \mathrm{S}}[1], \ldots, R_{\mathrm{w}, \mathrm{s}}[N-1]\right]^{\mathrm{T}}$ defines the DFT results with the windowing for the $s$-th sub-band and $(\cdot)^{\mathrm{T}}$ is the transpose. Through the switch block, signal-based spectrum results are stored in an efficient memory block with size $N \times M$.

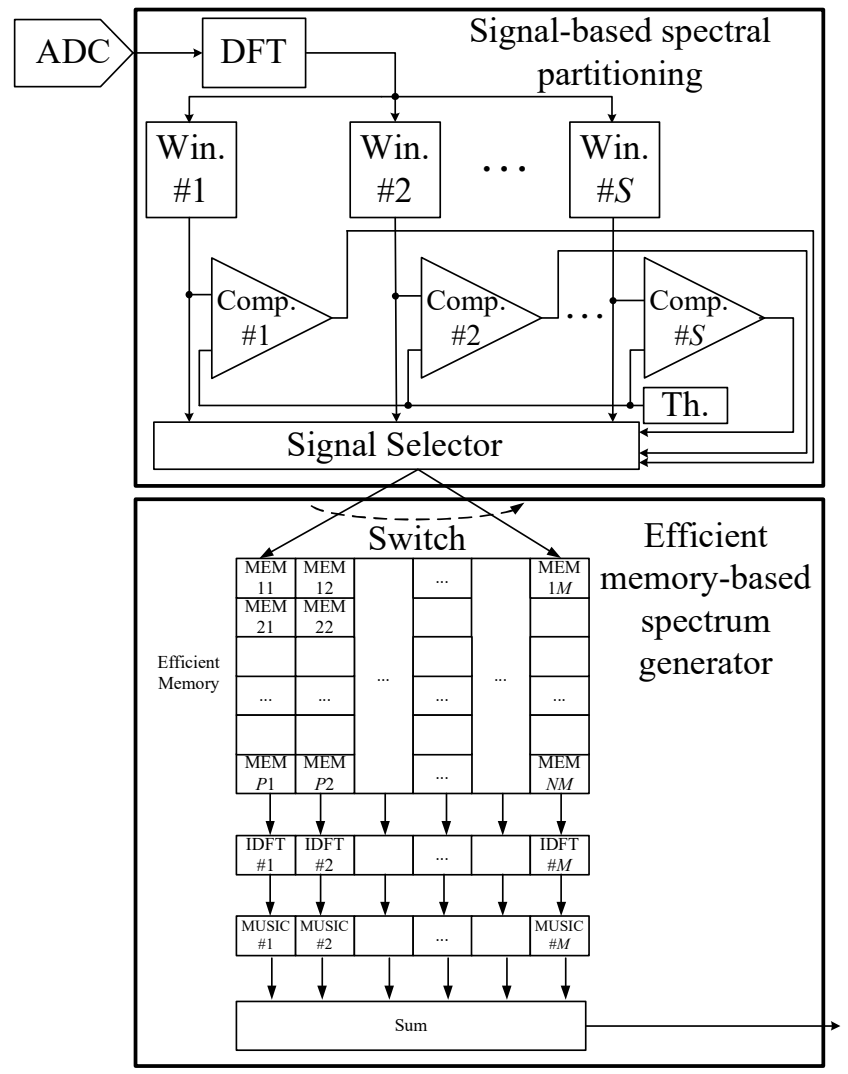

Fig. 1. Block diagram of proposed algorithm based on low complexity SPMUSIC.

\section{B. Efficient Memory-based Spectrum Generator Method}

Using windowing DFT results chosen by the signal selector block and storing at the memory block, the inverse DFT (IDFT) block for the $s$-th sub-band is achieved such as 


$$
r_{w, S}[n]=\sum_{l=0}^{N-1} R_{w, s}[l] e^{j 2 \pi n l / N} \text { for } \mathrm{n}=0,1, \ldots, N-1,
$$

where $r_{w, s}[n]$ is stored at the memory with indexes $s$ and $n$. For example, $r_{w, 0}[0]$ and $r_{w, 1}[1]$ are located at MEM00 and MEM11, respectively. The MUSIC method is then processed using the signal $r_{w, s}[n]$ with reduced noise power and $N$ samples. The autocorrelation matrix $\boldsymbol{R}_{\mathrm{s}}$ of $N$ by $N$ is defined as

$$
\mathbf{R}_{\mathrm{s}}=\sum_{n=0}^{N-1} \mathbf{r}_{\mathrm{w}, \mathrm{s}} \mathbf{r}_{\mathrm{w}, \mathrm{S}}^{\mathrm{H}},
$$

where the sequence $\mathbf{r}_{\mathrm{w}, \mathrm{s}}=\left[r_{\mathrm{w}, \mathrm{s}}[0], r_{\mathrm{w}, \mathrm{S}}[1], \ldots, r_{\mathrm{w}, \mathrm{s}}[N-1]\right]^{\mathrm{T}}$. The eigenvalue decomposition (EVD) of $\mathbf{R}_{\mathrm{s}}$ has a form given by

$$
\mathbf{R}_{\mathrm{S}}=\left[\begin{array}{ll}
\mathbf{E}_{\mathrm{S}} & \mathbf{E}_{\mathrm{N}}
\end{array}\right]\left[\begin{array}{cccc}
\lambda_{0} & 0 & \vdots & 0 \\
0 & \lambda_{1} & \ddots & \vdots \\
\vdots & \ddots & \ddots & 0 \\
0 & \cdots & 0 & \lambda_{N-1}
\end{array}\right]\left[\begin{array}{c}
\mathbf{E}_{\mathrm{S}}^{*} \\
\mathbf{E}_{\mathrm{N}}^{*}
\end{array}\right]
$$

where the signal eigenvector matrix $\mathbf{E}_{\mathrm{S}}=\left[\boldsymbol{e}_{0}, \ldots, \boldsymbol{e}_{M-1}\right]$ contains $M$ eigenvectors that span the signal subspace of the correlation matrix, the noise eigenvector matrix $\mathbf{E}_{\mathrm{N}}=\left[\boldsymbol{e}_{M}, \ldots\right.$ , $\left.\boldsymbol{g}_{N-1}\right]$ indicates $N-M$ eigenvectors spanning the noise subspace of the correlation matrix, and $\lambda_{n}$ denotes the $n$-th eigenvalues of $\mathbf{R}_{\mathrm{s}}$. The largest $M$ eigenvalues of $\lambda_{0}, \ldots, \lambda_{M-1}$ correspond to the $M$ eigenvectors of $\mathbf{E}_{S}$. The other eigenvalues $\lambda_{M}, \ldots, \lambda_{N-1}$ correspond to the eigenvectors of $\mathbf{E}_{N}$ such that $\lambda_{M}=\ldots=\lambda_{N-1}=\sigma^{2}$. Then, according to [5], the frequency spectrum of the noise-reduced signal for $s$-th subband is expressed as

$$
s_{S}(\tau)=\frac{1}{a^{H}(\tau) \mathbf{E}_{N} \mathbf{E}_{N}^{H} a(\tau)}
$$

where $a(\tau)$ is defined by $a(\tau)=\left[1, e^{j \mu \tau T_{\mathrm{s}}}, \ldots, e^{j(N-1) \mu \tau T_{\mathrm{s}}}\right]^{\mathrm{T}}$. After all the MUSIC results based on SP are summed, we can finally obtain the time delays $\tau$ of $M$ targets to find the peak finding in the proposed spectrum.

\section{Simulation Results}

In order to evaluate the performance of the proposed algorithm, we achieve a snapshot of the range spectrum and a Monte-Carlo simulation averaged over 10,000 estimates. In the following simulations, we set the parameters as shown in Table I.

TABLE I. SIMULATION PARAMETERS FOR AWGN CHANNEL.

\begin{tabular}{|c|c|}
\hline Parameter & Value \\
\hline$\mu$ & $2.5 \times 10^{12}$ \\
\hline$T_{\mathrm{s}}$ & $200 \mathrm{~ns}$ \\
\hline$T_{\mathrm{sym}}$ & $80 \mu \mathrm{s}$ \\
\hline
\end{tabular}

With $1.5 \mathrm{~m}$ distance resolution related with $1 /$ bandwidth, when the two targets were placed at $R_{1}=5 \mathrm{~m}$ and $R_{2}=$
$5.8 \mathrm{~m}$, respectively, the range spectra for DFT, MUSIC, and the proposed method were derived as shown according to different SNR in Fig. 2 and Fig. 3, respectively. In Fig. 2, in the case of $\mathrm{SNR}=-22 \mathrm{~dB}$ and single target, peaks of the DFT and the proposed algorithm were observable. However, the detected peak of the MUSIC does not exist compared with the real value due to the low SNR. In Fig. 3, in the case of SNR $=10 \mathrm{~dB}$ and two targets, two peaks of DFT and MUSIC were observable but not sharp. However, the detected peaks of the proposed algorithm are sharper than the peaks of the other conventional algorithm and closest to the real value. This characteristic is found in the measured results as well, as shown in Section V.

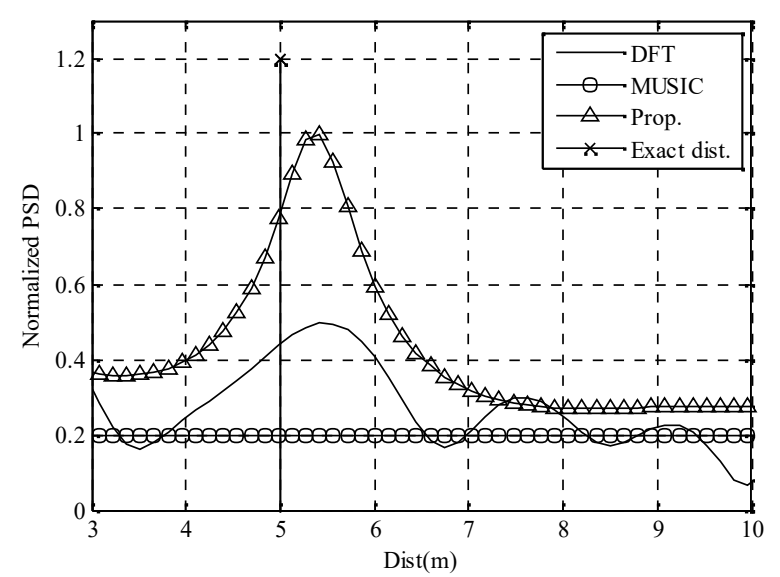

Fig. 2. Range spectrum of various algorithms for single target at $\mathrm{SNR}=$ $22 \mathrm{~dB}$.

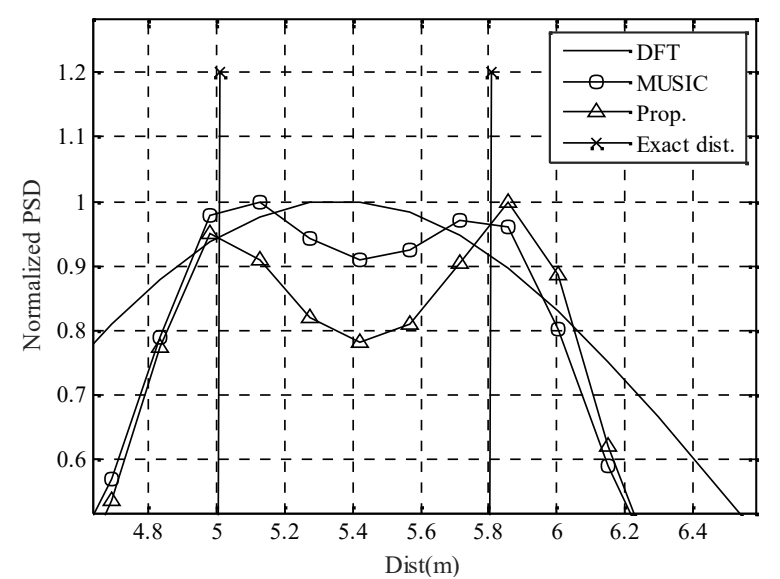

Fig. 3. Range spectrum of various algorithms for two targets at SNR = $10 \mathrm{~dB}$.

In Fig. 4, the proposed algorithm is performed 10,000 times for two equal amplitude signals spaced with a range distance $\Delta R$ at each SNR. For the proposed and conventional algorithms, the probabilities of detection are defined by whether the spectrum can classify two targets. Here, $\Delta R$ was $80 \mathrm{~cm}$, which corresponds to a $2.6 \mathrm{~ns}$ time delay. Based on Fig. 4, to resolve two targets separated by $75 \mathrm{~cm}$ at a $P_{D}$ of 0.8 , the required SNR of the proposed algorithm is $11.5 \mathrm{~dB}$ for the simulated echo signals while that of the conventional MUSIC algorithm is $12.25 \mathrm{~dB}$ and the DFT cannot detect any targets. Due to this difference in the required SNR, we can obtain higher maximum distance to detect targets. Figure 5 shows the frequency root mean square error (RMSE) as a function of the SNR with $\Delta R=$ $80 \mathrm{~cm}$. As expected, it can be seen that the RMSE 
continuously decreases with an increasing SNR. In addition, the proposed algorithm gives the best result whereas DFT and MUSIC cannot estimate the time delays well when the SNR is small because of their poor performance.

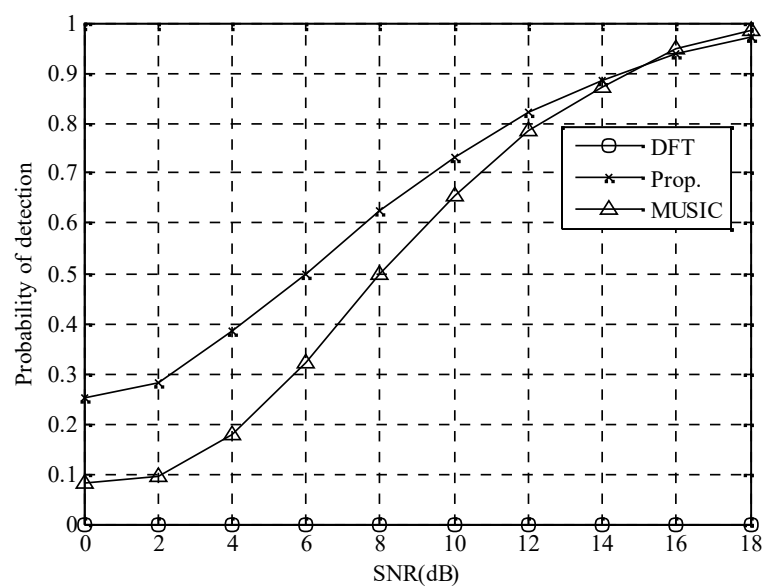

Fig. 4. Detection probability of various algorithms according to SNR.

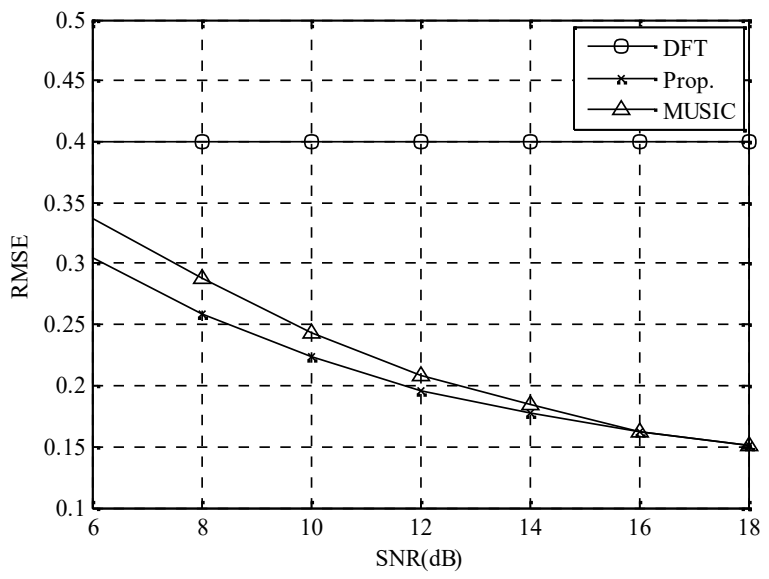

Fig. 5. RMSEs of various algorithms according to SNR.

\section{COMPUTATIONAL ANALYSIS}

The computational analysis evaluates the required processing time in the proposed algorithm compared with the conventional algorithm. We implemented various algorithms such as DFT, IDFT, and MUSIC in Cprograming with EVD and peak searching in the pseudo spectrum. The analysis parameters are as follows. The maximum distance is $300 \mathrm{~m}$ and the number of sub-bands is 10. The proposed method reduces the processing time by more than $89 \%$ with two objects, whereas the processing time with the conventional SP-MUSIC was constant irrespective of the number of objects. We evaluated the developed algorithms with the MATLAB program running at $2.4 \mathrm{GHz}$ with an Intel processor. The processing time for each of the implemented algorithms was measured by CPUTIME of MATLAB through the start-time and the endtime of the processor. The FMCW chirp parameters used in section IV for simulations are applied for the computation analysis in the same manner. The executed processing times for the implemented time delay estimation algorithms are summarized in Table II. The conventional SP based MUSIC has $\mathrm{S}$ times of hardware complexity compared with general MUSIC. The complexity of proposed method is similar with that of MUSIC algorithm.
TABLE II. PROCESSING TIME

\begin{tabular}{|c|c|}
\hline Algorithm & Processing Time \\
\hline Proposed & $0.64 \mathrm{~s}$ \\
\hline MUSIC & $0.56 \mathrm{~s}$ \\
\hline SP-MUSIC & $5.44 \mathrm{~s}$ \\
\hline
\end{tabular}

\section{EXPERIMENTS}

To verify the performance of the proposed method in a real environment, we performed a variety of experiments inside an anechoic chamber located at Daegu-Gyeongbuk Institute of Science \& Technology (DGIST) in South Korea [3]. The chamber was built for wireless signals of $8 \mathrm{GHz}$ to $110 \mathrm{GHz}$. The dimensions are $10 \mathrm{~m}(\mathrm{~L}) \times 5 \mathrm{~m}(\mathrm{~W}) \times 4 \mathrm{~m}$ (H). As shown in [3], a maximum of four targets, which could be moved back and forth, were used for our experiments. By using the chamber environment, we can find the exact positions of the targets to assess the algorithm performance.

\section{A. RF Transceiver}

A block diagram of the $24 \mathrm{GHz}$ FMCW radar system is shown in Fig. 6. The system comprises a single transmitter, a single transmitting antenna, two receiving antennas, and two receiving channels. The transmitter generates FMCW signals. The initial transmission time of each sweep is determined by a signal processing board. The received signal from the receiving antennas is used to process the proposed algorithm. The transmitter contains a voltagecontrolled oscillator (VCO), a frequency synthesizer, and a $26 \mathrm{MHz}$ oscillator. To generate the FMCW source, the frequency synthesizer controls the input voltage of the VCO. The source sweeps over a range of $24.025 \mathrm{GHz}-24.225 \mathrm{GHz}$ with $8 \mathrm{dBm}$ output power. The transmitted RF signal is sent to the transmitted antenna and receiver mixer via the power divider.

The receiver comprises three LNAs, three mixers, two high-pass filters (HPFs), and two low-pass filters (LPFs). The receiver has an overall noise figure of $8 \mathrm{~dB}$. The gain and noise figure of the LNA are $14 \mathrm{~dB}$ and $2.5 \mathrm{~dB}$, respectively. The received $\mathrm{RF}$ signal is down-converted to an IF signal (beat signal) by the mixer. The measured $3 \mathrm{~dB}$ cutoff frequency of the HPF and the LPF is about $13 \mathrm{KHz}$ and $2 \mathrm{MHz}$, respectively.

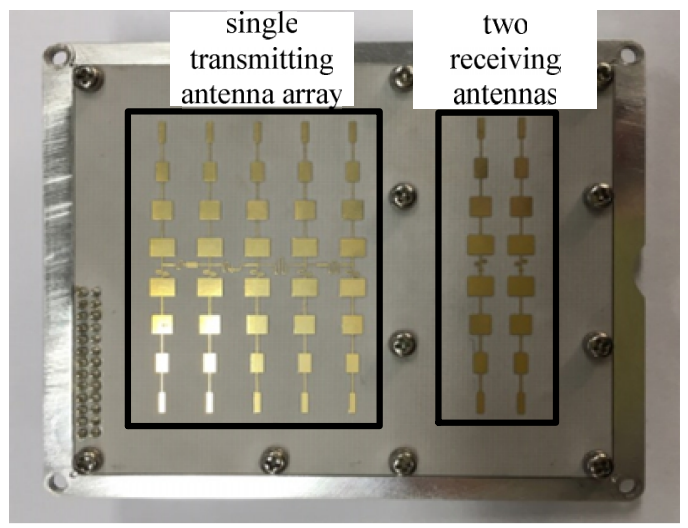

Fig. 6. Photograph of the $24 \mathrm{GHz}$ FMCW radar system.

\section{B. Experimental Procedure}

We conducted an experiment with the proposed method 
for a single target in the chamber.

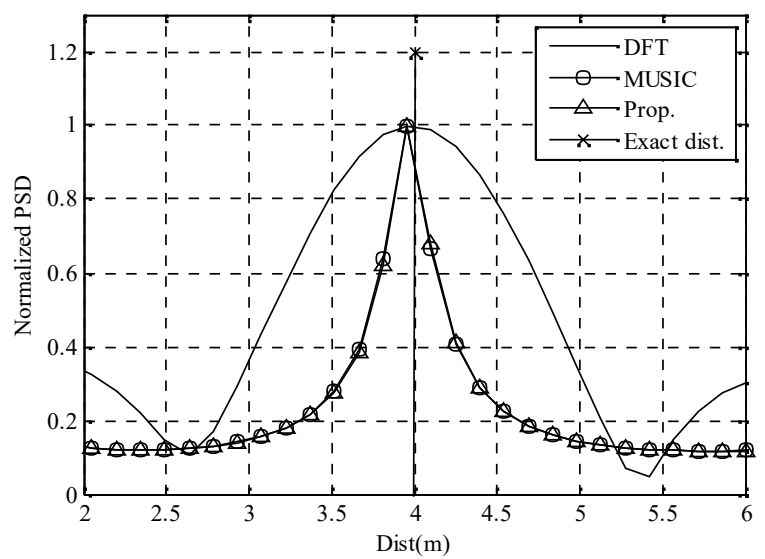

(a)

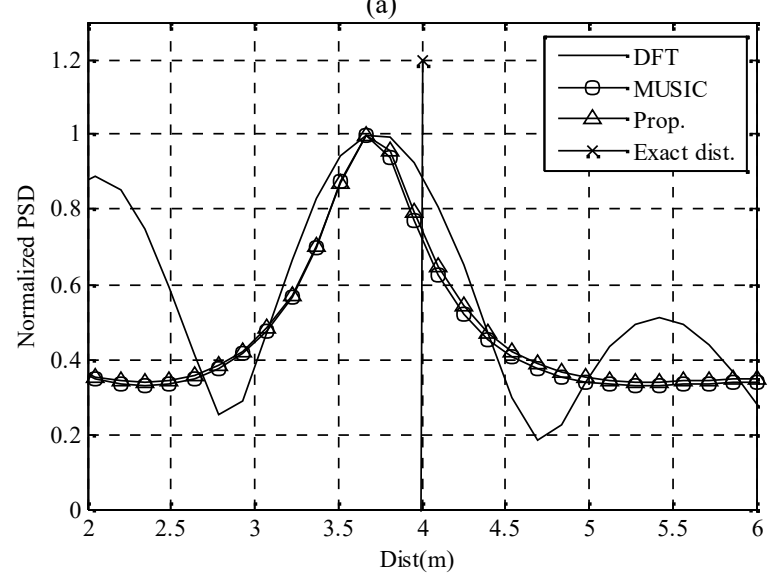

(b)

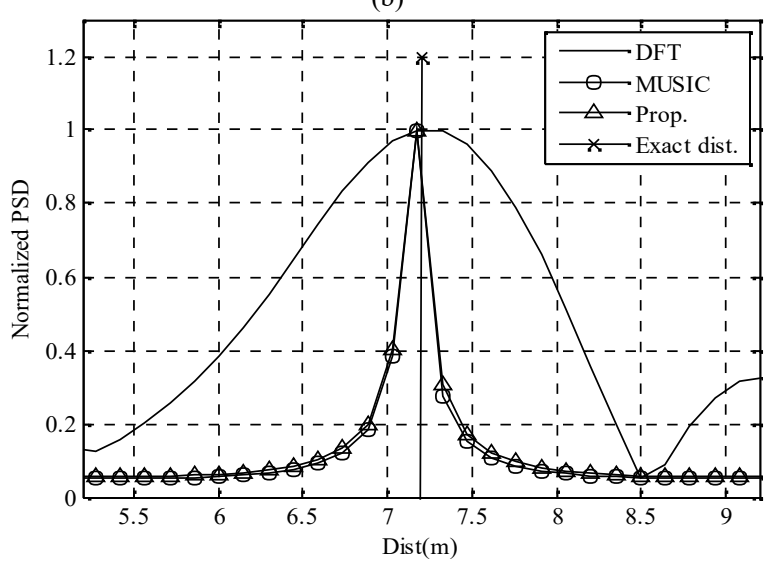

(c)

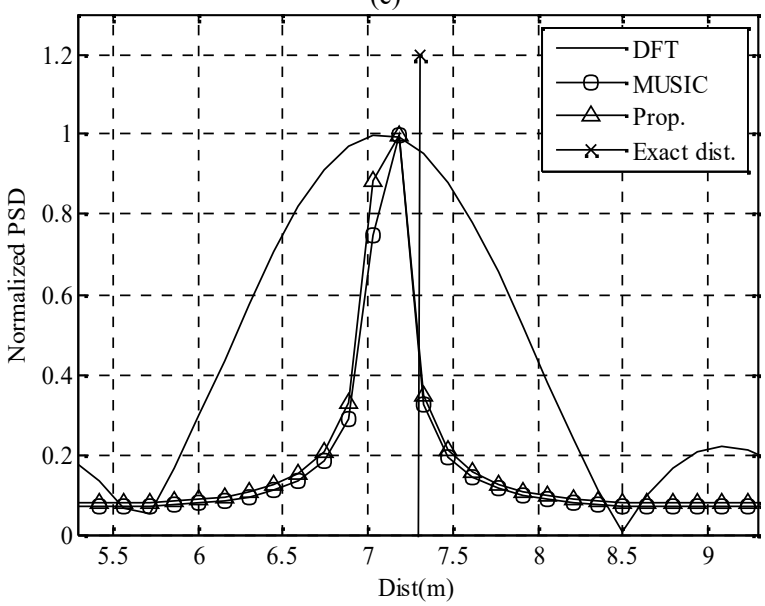

(d)

Fig. 7. Experimental results: a) $\left[R_{1}, \theta_{1}\right]=\left[4.2 \mathrm{~m},-0^{\circ}\right]$, b) $\left[R_{2}, \theta_{2}\right]=[4.4 \mathrm{~m}$, $\left.-30^{\circ}\right]$, c) $\left.\left[R_{3}, \theta_{3}\right]=\left[7.2 \mathrm{~m},-3^{\circ}\right], \mathrm{d}\right)\left[R_{4}, \theta_{4}\right]=\left[7.3 \mathrm{~m},-30^{\circ}\right]$.
In order to guarantee the validity of the proposed algorithm in a variety of situations, the range map was derived as given at $\left[R_{1}, \theta_{1}\right]=\left[4.2 \mathrm{~m},-0^{\circ}\right],\left[R_{2}, \theta_{2}\right]=[4.4 \mathrm{~m},-$ $\left.30^{\circ}\right],\left[R_{3}, \theta_{3}\right]=\left[7.2 \mathrm{~m},-3^{\circ}\right]$ and $\left[R_{4}, \theta_{4}\right]=\left[7.3 \mathrm{~m},-30^{\circ}\right]$ in the anechoic chamber in Fig. 7.

As seen in Fig. 7, the proposed and the conventional SPMUSIC method could accurately estimate the range spectrum. However, SP-MUSIC has higher complexity than the proposed algorithm because it chooses all regions of the DFT.

\section{DISCUSSION}

The proposed SP-MUSIC with low complexity has similar range resolution performance compared to conventional MUSIC-based high-resolution algorithm, but has significantly lower processing time performance. Especially, in the low SNR environment, the performance of the proposed algorithm is improved compared to the conventional MUSIC algorithm in single target and multiple targets. It is expected to improve the parameter performance of the vehicle radar by implementing the proposed algorithm composed of the conventional FFT or MUSIC in the embedded system of automotive radar.

\section{CONCLUSIONS}

We have proposed the low complexity SP-MUSIC algorithm for automotive radar. To improve the performance of range estimation, high resolution based algorithms such as the ESPRIT and MUSIC have been proposed. However, in a low SNR, the high resolution algorithm shows degraded performance to estimate the parameters. To solve this problem, SP based high resolution algorithms have been studied recently. However, for real-time operation, the conventional SP method cannot be applied to automotive radar due to its high complexity. So, we need to propose the low complexity high resolution algorithms. The proposed method consists of DFT, windowing, comparator with a switch, memory, IDFT and MUSIC to satisfy the required accuracy requirements and reduce the complexity compared with the conventional SP-MUSIC estimator. The time delay performance of the proposed method is improved for various multi-targets in the presence of an AWGN channel. While the complexity of the proposed algorithm is less than that of the conventional algorithm, e. g. MUSIC, as determined through the Monte-Carlo simulation results and experimental results, the range resolution of the proposed method is similar to that of SP-MUSIC for various parameters. The proposed method is applicable to vehicle radar due to its low complexity characteristics.

\section{REFERENCES}

[1] M. M. Reja, Z. Hameed, K. Moez, S. Shamsadini, "Compact CMOS IR-UWB transmitter using variable-order Gaussian pulse generator", Electronics Letters, vol. 49, no. 16, 2013. [Online]. Available: http://dx.doi.org/10.1049/el.2013.1975

[2] K. Sobaihi, A. Hammoudeh, D. Scammell, "Field-programmable gate array-based software-defined radio for millimetre-wave single-carrier transmission", IET Networks, vol. 1, no. 4, pp. 239-248, 2012. [Online]. Available: http://dx.doi.org/10.1049/iet-net.2012.0037

[3] S. Kim, D. Oh, J. Lee, "Joint DFT-ESPRIT estimation for TOA and DOA in vehicle FMCW radars", IEEE Antennas and Wireless 
Propag. Lett., vol. 14, pp. 1710-1713, 2015. [Online]. Available: http://dx.doi.org/10.1109/LAWP.2015.2420579

[4] L. Qu, Q. Sun, T. Yang, L. Zhang, Y. Sun, “Time-delay estimation for ground penetrating radar using ESPRIT with improved spatial smoothing technique", IEEE Geosci. Remote Sens. Lett., vol. 11, no. 8, pp. 1315-1319, 2014. [Online]. Available: http://dx.doi.org/10.1109/LGRS.2013.2292825

[5] L. Sun, Y. Li, H. Hong, F. Xi, W. Cai, X. Zhu, "Super-resolution spectral estimation in short-time non-contact vital sign measurement”, Rev. Sci. Instrum., vol. 86, 2015. [Online]. Available: http://dx.doi.org/10.1063/1.4916954

[6] B. W. Choi, E. H. Bae, J. S. Kim, K. K. Lee, "Improved prewhitening method for linear frequency modulation reverberation using dechirping transformation", Journal of the Acoustical Society of America, vol. 123, no. 3, 2008. [Online]. Available: http://dx.doi.org/ $10.1121 / 1.2838249$

[7] V. K. Nguyen, M. D. E. Turley, G. A. Fabrizio, "A new data extrapolation approach based on spectral partitioning", IEEE signal Proess. Lett., vol. 23, no. 4, pp. 454-458, 2016. [Online]. Available: http://dx.doi.org/10.1109/LSP.2016.2533602. 